\title{
Active play key to curbing child obesity
}

$\mathrm{C}$ anada is a world leader when it comes to public spaces and programs for physical activity. Nine out of 10 families live near parks and other facilities like pools or arenas. Four out of five kids regularly participate in organized sport. And all provinces and territories have mandatory physical education in schools. So why are young Canadians sitting more, moving less and getting heavier?

About a third of Canadian kids were overweight or obese at last count in 2011, according to Statistics Canada. And the average child now burns 100 fewer calories per day than her counterpart did four decades ago, when just $15 \%$ of kids were obese.

Parents and policy-makers have looked to schools and organized sports to close this "energy gap." But there's growing evidence that these structured programs have little impact on kids' overall physical activity or body mass index, according to a 2015 article in the Canadian Journal of Diabetes.

"If you actually understand how much energy children expend doing these activities, you start to understand why these interventions are ineffective," said Ian Janssen, Canada Research Chair in Physical Activity and Obesity, at the Canadian Obesity Summit in Toronto. In a May 2 plenary, Janssen argued that kids have the potential to burn far more energy in unstructured, active play - in other words, by simply "letting kids go outside and be kids."

By Janssen's calculations, the average 11-year-old can burn an extra 49 calories per day by replacing a single hour of screen time with active play. That's more energy than the same child would expend to meet national benchmarks for organized sport (23 calories per day), walking or biking to school (16 calories per day) and physical education (6 calories per day) combined. If these benchmarks seem low, it's because the energy you burn depends in part on body weight.

Studies usually don't discuss the intensity of movement involved in struc-

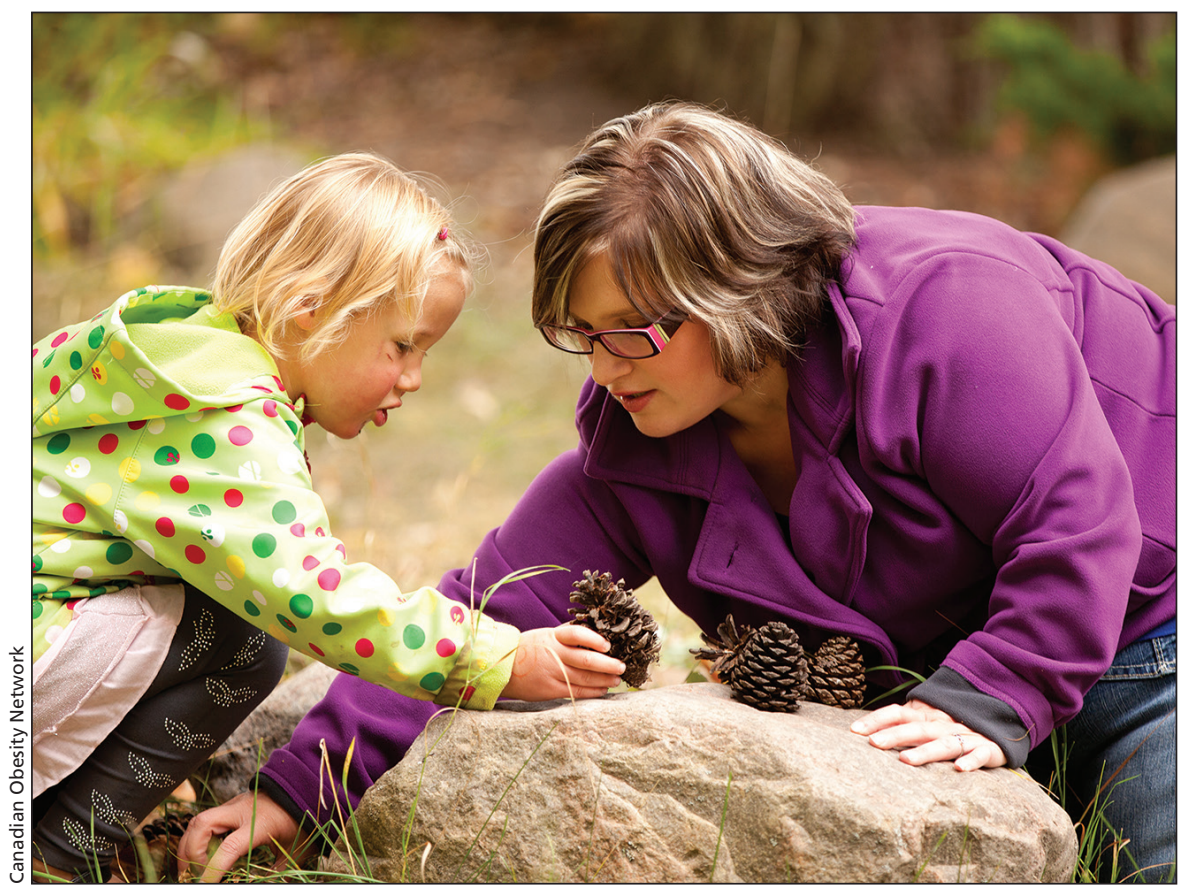

Unstructured play can burn more calories than school and sports programs combined, says Canada Research Chair in Physical Activity and Obesity lan Janssen.

tured interventions, but "the amount of actual activity is low," explained Janssen. Accelerometer data indicate that kids are sedentary for three-quarters of the time they spend in physical education class and one-third of the time they spend playing sports.

Averaged out over the year, the daily time commitment required to meet benchmarks is also low, amounting to just 16 minutes of physical education and 22 minutes of organized sport per day.

Meanwhile, the average 11-year-old spends 5.8 hours of their free time each day sitting in front of screens. This has largely been carved out from time kids used to spend in active play, said Janssen. "For many kids this is what they now spend their entire weekends doing."

Unstructured playtime isn't particularly intense - about half is spent sedentary — "but it's the volume that's different," said Janssen. Restricting screen time to a maximum two hours per day frees up nearly four hours for active play. Taking intensity into account, an average 11-year-old could be burning an extra 186 calories every day, or the equivalent of a candy bar.

Boosting Canada's current $\mathrm{F}$ grade for active play to a D by getting $20 \%$ of kids to this benchmark would address $37 \%$ of the current "energy gap," said Janssen. In comparison, improving Canada's already high grades for organized sport and physical education would close just $2 \%$ of the gap.

Encouraging playtime away from screens may also be more a more realistic strategy for kids who are intimidated by organized activities, added Janssen. "When they get into sport, there are all kinds of rules and pressure and there are a lot of dropouts."

Active play has the further advantage of being free, he said. "You don't need to spend money or drive your kid to a special place; you just open your front door."

According to Janssen, the most successful interventions acknowledge that there's limited opportunity to increase physical activity during the school day. 
Those like the Alberta Project Promoting active Living and healthy Eating (APPLE Schools) include activities led by students outside school, such as planting gardens and cooking classes. Some schools also open their gymnasiums after hours to give kids a safe space for active play.

Paul Veugelers, a public health professor at the University of Alberta in Edmonton, based the APPLE model on a similar program in Nova Scotia. "Kids in these schools reported healthier diets, more physical activity, less screen time, and most impressively $60 \%$ less overweight and $70 \%$ less obesity compared to other students," Veugelers told participants in a May 1 plenary.

Alberta has seen similar results since rolling out the project to 51 schools in disadvantaged communities. Curiously, most of the program's positive impact on physical activity has come from kids moving more in their free time, said Veugelers. "This may be why complex programs are more successful than those with a single target [like getting kids involved in organized sport] because the kids are more likely to take the message and practise it when they're away from school." - Lauren Vogel, CMAJ

CMAJ 2015. DOI:10.1503/cmaj.109-5058 